\author{
MITSUBISHI ELECTRIC RESEARCH LABORATORIES \\ http://www.merl.com
}

\title{
Spectral Shaping of UWB Signals for Time-Hopping Impulse Radio
}

\author{
Y-P Nakache, A.F. Molisch
}

TR2005-120 April 2005

\begin{abstract}
This paper studies the design of signaling waveforms for time-hopping impulse radio (TH-IR) with limits on the power spectral density. Such restrictions are imposed by the spectral mask prescribed by frequency regulators for ultrawideband (UWB) signals. The "conventional" THIR system with pulse-position modulation and time-hopping multiple access gives rise to spectral lines that either violate the regulations, or require a significant power back-off. To remedy this situation, we propose the use of polarity randomization, which eliminates the spectral lines and also leads to a smoothing of the continuous part of the spectrum. We analyze different variants of the polarity randomization, considering short and long randomization sequences, as well as symbol-based or pulse-based randomization. We analyze the effect of this technique on both PPM and BPSK modulation.
\end{abstract}

IEEE Journal on Selected Areas in Communications

This work may not be copied or reproduced in whole or in part for any commercial purpose. Permission to copy in whole or in part without payment of fee is granted for nonprofit educational and research purposes provided that all such whole or partial copies include the following: a notice that such copying is by permission of Mitsubishi Electric Research Laboratories, Inc.; an acknowledgment of the authors and individual contributions to the work; and all applicable portions of the copyright notice. Copying, reproduction, or republishing for any other purpose shall require a license with payment of fee to Mitsubishi Electric Research Laboratories, Inc. All rights reserved.

Copyright (C) Mitsubishi Electric Research Laboratories, Inc., 2005

201 Broadway, Cambridge, Massachusetts 02139 



\title{
Spectral Shaping of UWB Signals for Time-Hopping Impulse Radio ${ }^{1}$
}

\author{
Yves-Paul Nakache ${ }^{2}$, Member, IEEE, and Andreas F. Molisch, Fellow, IEEE
}

\begin{abstract}
This paper studies the design of signaling waveforms for time-hopping impulse radio (TH-IR) with limits on the power spectral density. Such restrictions are imposed by the spectral mask prescribed by frequency regulators for ultrawideband (UWB) signals. The "conventional" TH-IR system with pulseposition modulation and time-hopping multiple access gives rise to spectral lines that either violate the regulations, or require a significant power back-off. To remedy this situation, we propose the use of polarity randomization, which eliminates the spectral lines and also leads to a smoothing of the continuous part of the spectrum. We analyze different variants of the polarity randomization, considering short and long randomization sequences, as well as symbol-based or pulse-based randomization. We analyze the effect of this technique on both PPM and BPSK modulation.
\end{abstract}

\section{Introduction}

UWB communications first received attention in the 1990s, due to the groundbreaking work of Win and Scholtz [1], [2], [3]. They proposed to use time-hopping impulse radio (TH-IR) as multiple access method, combined with pulse position modulation (PPM). In the TH-IR scheme, each symbol is represented by a sequence of pulses, where the delay between the pulses is determined by a pseudorandom sequence; multiple-access (MA) capability is provided by using different TH sequences for different users. The use of short pulses in the transmit signal leads to a wide spreading of the spectrum. While alternative UWB schemes have been developed [4], [5], TH-IR has remained the method of choice for most of the academic, as well as a lot of industrial work; for an overview, see [6], [7], [8].

A keystone in the development of UWB was the 2002 "Report and Order" issued by the frequency regulator in the USA, the Federal Communications Commission (FCC), which allowed the use of unlicensed UWB transmission between 3.1 and $10.6 \mathrm{GHz}$, subject to the fulfillment of a spectral emission mask that limits the power spectral density (PSD) within each 1 $\mathrm{MHz}$ band to $-41.3 \mathrm{dBm} / \mathrm{MHz}$ [9], [10]. This ruling had important consequences for the design of UWB signals. Due to the low total allowed PSD, it is important that the spectrum of the transmit signal exploits the mask as well as possible. Signals that cause spectral lines are highly undesirable, as they have a high peak PSD. Thus, those signals have to back off the average power until the peak of the spectrum complies with the mask.

The analysis of the spectral properties of linear modulation formats and PPM (without time hopping) is well documented in the literature (e.g., [11],[12]). The study of the spectral

\footnotetext{
1 Part of this work was presented at VTC spring 2003

2 Author for correspondence (e-mail: nakachey@merl.com)
}

properties of TH-IR combined with those modulation formats, however, is fairly recent. [2] considered some fundamental properties of the spectrum of "classical" TH-IR, while [13] and [14] analyzed the impact of timing jitter on such systems. Piazzo and Romme analyzed the PSD further in [15] and suggested a way to control the spectrum by means of TH codes [16]. The use of polarity randomization for TH-IR was first suggested by us in 2003 [17]. Cassioli and Mazzenga [18] derived a general expression for the PSD of PPM signals with TH and DSC (Delay Coded Sequence). Maggio et al. [19] proposed a pseudo chaotic time hopping scheme to enhance the spectral characteristic of the system, trying to remove periodic structures from the signal. It is also worth noticing that parallels can be drawn from the analysis of direct-sequence CDMA signals; the spectrum of DS-CDMA is discussed, e.g., in [20]. A study of spectral shaping using code-hopping CDMA was written in 2004 by A. Saidi [21].

The classical TH-IR leads to spectral lines, and is thus not well suited for operation according to the FCC regulations. In this paper, we propose a modification that eliminates the spectral lines, and shows in general better spectral properties. This scheme is based on a randomization of the polarity of the transmitted pulses. This scheme can be used in conjunction with either PPM or BPSK (binary phase shift keying) modulation and different types of TH sequences.

The key contributions of our paper are thus the following:

- We propose a new method that eliminates the spectral lines of TH-IR-PPM, and improves the spectral properties of THIR. This suggestion (first presented by us at a conference in 2003 [17]) has since been widely accepted and also impacted various proposals for the IEEE 802.15.4a standard that is currently under development.

- Using the well known theory of power spectrum analysis, we now quantitatively detail the impact of this method when used for different types of time-hopping sequences, including short hopping sequences (sequence length equal to the symbol length), long hopping sequences, as well as randomization on a symbol-by-symbol basis.

- We investigate the impact of the frame structure of TH-IR on the spectra of our method.

The remainder of the paper is organized in the following manner: Section 2 gives analytical formulations for the spectrum of "conventional" TH-PPM, showing the spectral lines created by this MA/modulation scheme, and summarizes the most relevant requirements of the FCC regulations. Section 3 introduces the symbol-based polarity randomization which eliminates the spectral lines. Section 4 presents the pulse-based polarity randomization, which solves the problem of the spectral crest factor due to the structure of the TH sequence. A summary and conclusions wrap up this paper.

Yves-Paul Nakache and Andreas Molisch are with Mitsubishi Electric Research Labs, Cambridge, MA, USA

Andreas Molisch is also with the Department of Electroscience, Lund University, Lund, Sweden

(e-mail: Andreas.Molisch@ieee.org) 


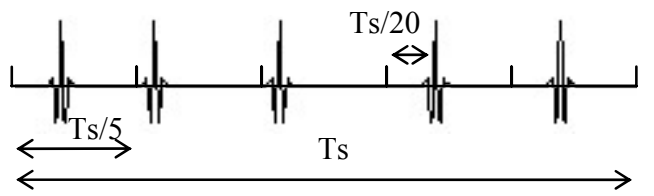

Fig. 1: TH structure of sequence [ [ $\left.\begin{array}{lllll}1 & 0 & 0 & 1 & 1\end{array}\right]$

The results presented in this paper were verified by theoretical analysis and Matlab simulations. In order to present clear illustrations of the results, a bandwidth of $4 \mathrm{GHz}$ (frequency range 3-7 GHz) was used to display the PSDs and their spectral crest factor. However, the spectrum of our waveforms also fits the FCC mask at frequencies $>7 \mathrm{GHz}$ as shown in [17]. The parameters for the examples are chosen such that they are suitable for a high-data-rate system $(100$ $\mathrm{Mbit} / \mathrm{s})$. However, we stress that the basic approach is equally valid for other data rates. In particular, it can be applied to lowdata rate systems as envisioned, e.g., for the IEEE 802.15.4a standard.

\section{Spectral characteristics of classical TH-IR}

\section{II.A Transmit waveform of TH-IR}

In this section, we review and re-interpret the spectral characteristics of classical TH-IR. Fig. 1 shows the structure of the transmit signal. The transmit signal is given as $[2,3]$

$s_{t r}(t)=\sum_{j=-\infty}^{\infty} x\left(t-j T_{f}-c_{j} T_{c}-b_{\left[j / N_{f}\right\rfloor} \Delta\right)$

where $x(t)$ is the transmitted constituent pulse, $T_{f}$ is the average pulse repetition time, $N_{f}$ is the number of frames (and therefore also the number of pulses $N_{s}$ ) representing one information symbol of length $T_{s}$, and $b$ is the information symbol transmitted. $\Delta$ is the time shift of the PPM.

The TH sequence provides an additional time shift of $c_{j} T_{c}$ seconds to the $j^{\text {th }}$ pulse of the signal, where $T_{c}$ is the chip interval, and $c_{j}$ are the elements of a pseudorandom sequence, taking on integer values between 0 and $N_{c}-1$. To prevent pulses from overlapping, the chip interval is selected to satisfy $T_{c} \leq T_{f} / N_{c}$; in the following, we assume $T_{f} / T_{c}=N_{c}$ so that $N_{c}$ is the number of chips per frame.

\section{II.B Power spectral density}

In this section, we review the pertinent details of the FCC regulations, as well as some basic equations for the computation of the PSD.

The FCC regulations provide limits to the PSD of the emissions, given in the form of a PSD mask. In the band from $3.1 \mathrm{GHz}$ to $10.6 \mathrm{GHz}$, the PSD is limited to $-41.3 \mathrm{dBm} / \mathrm{MHz}$. The limits on the PSD must be fulfilled for each possible $1 \mathrm{MHz}$ band, but not necessarily for smaller bandwidths. For these systems there is also a limit on the peak emission level contained within a $50 \mathrm{MHz}$ bandwidth centered on the frequency, $\mathrm{f}_{\mathrm{M}}$, at which the highest radiated emission occurs.

The FCC has adopted a limit on the (temporal) peak power, based on a sliding scale dependent on the actual resolution bandwidth (RBW) employed in the measurement. The peak EIRP limit is $20 \cdot \log (\mathrm{RBW} / 50) \mathrm{dBm}$ when measured with a resolution bandwidth RBW ranging from $1 \mathrm{MHz}$ to $50 \mathrm{MHz}$.

Only one peak measurement, centered on $f_{\mathrm{M}}$, is required. As a result, UWB emissions are average-power-limited for PRFs greater than $1 \mathrm{MHz}$ and peak-power limited for PRFs below 1

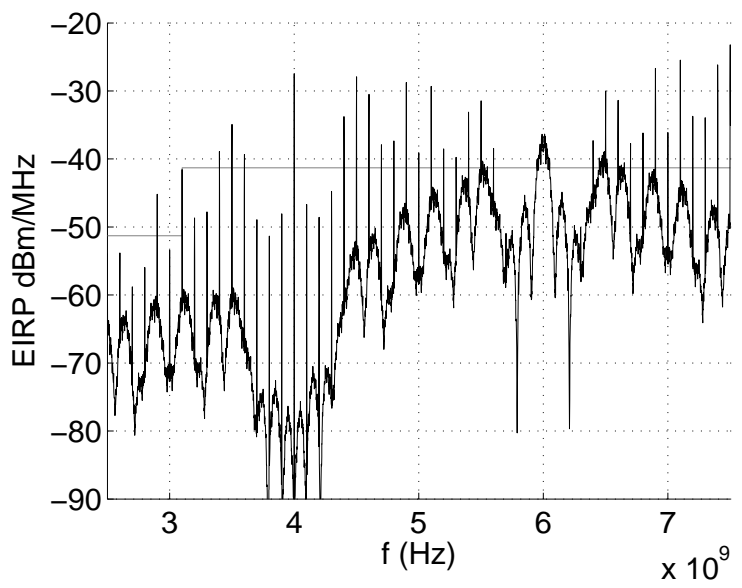

Fig. 2: PSD of TH-IR with 2-PPM

MHz. Since the latter case provides less energy per symbol at the receiver, PRFs above $1 \mathrm{MHz}$ should be used whenever possible.

The following equation for the PSD of M-ary modulation with independent input sequences [22] will be used extensively in the remainder of the paper.

$$
\begin{aligned}
& G_{s}(f)=\frac{1}{T_{s}^{2}} \cdot \sum_{n=-\infty}^{+\infty}\left(\left|\sum_{i=0}^{M-1} P_{i} \cdot S_{i}\left(\frac{n}{T_{s}}\right)\right|^{2} \delta\left(f-\frac{n}{T_{s}}\right)\right) \\
& +\frac{1}{T_{s}}\left(\sum_{i=0}^{M-1} P_{i} \cdot\left|S_{i}(f)\right|^{2}-\left|\sum_{i=0}^{M-1} P_{i} \cdot S_{i}(f)\right|^{2}\right)
\end{aligned}
$$

Here, $M$ denotes the size of the modulation alphabet, $T_{\mathrm{s}}$ the symbol period, $S_{i}(f)$ the Fourier transform of $s_{i}(t)$, i.e., the waveform of the $i^{\text {th }}$ symbol of the constellation and $P_{i}$ the marginal probability of the $i^{\text {th }}$ symbol.

\section{II.C. PSD of TH-IR with short TH sequence}

The PSD of the transmit signal is determined both by the pulse shape $s(t)$ and the time-hopping sequence $c_{j}$. A majority of systems considered in the literature and standardization working groups up to now assume that the duration of the $\mathrm{TH}$ sequence is identical to the symbol duration; similar to direct sequence CDMA, we henceforth refer to this case as "short" hopping sequence. Therefore, this TH sequence has a duration $\mathrm{T}_{\mathrm{s}}$ and it contains $\mathrm{N}_{\mathrm{s}}$ pulses. The spectrum of the transmit signal without modulation can be written as:

$F T\{T H(t)\}=X(f) \cdot \sum_{k=0}^{N_{s}-1} e^{j 2 \pi\left(T_{f} \cdot k+c_{k} T_{c}\right) \cdot f}$

The underlying pulse shape for the simulations of this paper is the fifth derivative of a Gaussian pulse, which was shown to be a good choice in [23]. $X(f)$ is its Fourier Transform. For most of the examples in the remainder of the text, we use a TH sequence with $\mathrm{N}_{\mathrm{s}}=5$ pulses following the sequence $c_{k}$ $[1,0,0,1,1]$, and 4 chips per frame (see Fig. 1), [24]. The number of pulses is a compromise between the requirements for a sufficient number of chips per frame, and the necessity of having a sufficient number of frames for each symbol. For a high data rate system, the symbol duration is limited to about $10 \mathrm{~ns}$, making $2 \mathrm{~ns}$ frame duration a reasonable compromise. Furthermore, a prime number like 5 helps to design a set of orthogonal TH sequences [28], [29], [30]. 
When 2-PPM is used as a modulation format, the PSD of the modulated signal can be shown to be [25], [31]:

$$
\begin{aligned}
& G_{s}(f)=\frac{1}{2 T_{s}^{2}} \sum_{n=-\infty}^{+\infty}\left|X\left(\frac{n}{T_{s}}\right)\right|^{2} \mid \sum_{k=0}^{\mid N_{s}-1} e^{\left.j 2 \pi\left(T_{f} \cdot k+c_{k} T_{c}\right) \cdot \frac{n}{T_{s}}\right|^{2}} \\
& +\frac{1}{2 T_{s}}|X(f)|^{2} \cdot\left|\sum_{k=0}^{N_{s}-1} e^{j 2 \pi\left(T_{f} \cdot k+c_{k} T_{c}\right) \cdot f}\right|^{2} \cdot\left(1-\cos \left(2 \pi n \Delta / T_{s}\right)\right) \cdot \delta\left(f-n / T_{s}\right)
\end{aligned}
$$

$\Delta$ is the delay due to the PPM; an example is shown in Fig.2.

The amplitude of these spectral lines can be $10 * \log 10\left(\mathrm{~T}_{\mathrm{s}}^{-1}\right)$ $\mathrm{dB}$ above the level of the continuous part of the spectrum, which corresponds to $80 \mathrm{~dB}$ for a $100 \mathrm{Mbps}$ data rate. In the context of FCC regulations, the regulations specify measurement procedures that average the power of these lines: over a $1 \mathrm{MHz}$ band. Therefore, these spectral lines are attenuated by a factor $60 \mathrm{~dB}$, as is also reflected in the figures above. Even in this case, the lines still remain about $20 \mathrm{~dB}$ above the continuous part of the spectrum (for $100 \mathrm{Mbps}$ data rate), and require a reduction of the total transmitted power of the signal by that amount. Uniform timing jitter, which is equivalent to considering a random position of the pulse within the frame, helps to smooth the spectrum but does not remove all spectral lines [15].

\section{D PSD of TH-IR with long TH sequence}

One method for improving the spectral properties is a further randomization of the position of the pulses, e.g., by using a long TH sequence. The goal behind the definition of a longer code is to average the PSD of different waveforms. If the peaks of their respective PSD occur at different frequencies, the total peak-to-average ratio of the PSD will be reduced. The same argument holds for the spectral lines: their averaged power will be lower.

The combination of long-code TH with PPM can then be seen as M-ary PPM, where the position of the pulse in each frame is completely random. This interpretation is strictly valid if the PPM shifts the position of a pulse in each frame cyclically, not linearly as is commonly assumed. However, for long frame durations, the difference between those two cases is minor. ${ }^{3}$ Fig. 3 shows an example where a symbol of 5 frames with 4 chips each is represented. The bit " 1 " modulates the signal by shifting the symbol by $\Delta$, which is equal to one chip duration. The last chip of the last frame contains a pulse if there is no modulation. With a linear shift of one chip duration due to the PPM, the pulse would be shifted outside its frame (and even outside the symbol). However, a cyclical shift moves the pulse to the first chip of the last frame. As a consequence, the probability of a pulse occurring at a specific chip is completely uniform even in the presence of PPM. ${ }^{4}$ A long TH sequence implies a randomly selected chip for the pulse position for each frame.

3 To simplify the derivation, a PPM shifting the symbol by a multiple of the chip duration is considered here, so that $\Delta=k \cdot T_{c}$.

4 an alternative approach, which is widely used, is a guard interval at the end of each frame. However, this approach leads to a non-uniform probability density function of the pulse position.
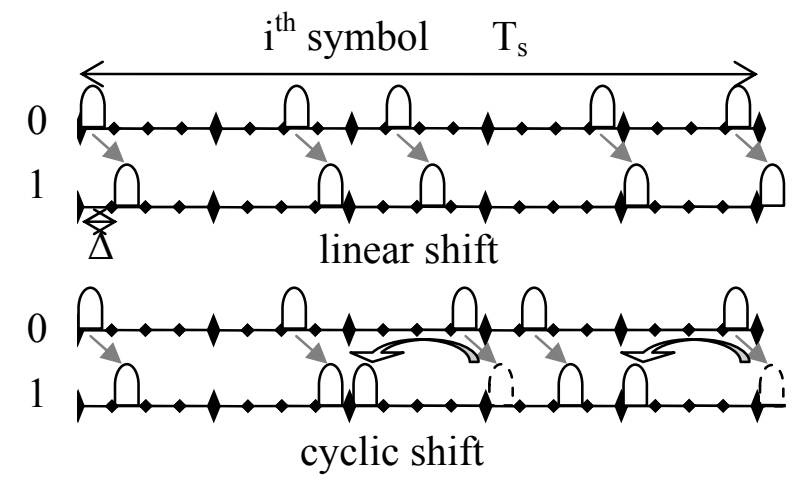

Fig. 3: linear shift versus cyclic shift of the pulse position sequence

Therefore the frames are independent and the pulse can have $\mathrm{M}$ equiprobable positions in each frame. The signal can be modeled as a M-ary PPM with $T_{f}$ as the symbol duration and $M=N_{c}$. The PSD can then be easily obtained from Eq. (2) with M-ary PPM modulation.

$G_{s}(f)=\frac{1}{N_{c}^{2} T_{f}^{2}} \cdot \sum_{n=-\infty}^{+\infty}\left(\left|\sum_{i=0}^{N_{c}-1} X\left(\frac{n}{T_{f}}\right) \cdot\left(e^{-j 2 \pi n T_{c}}\right)_{f}^{i}\right|^{2} \delta\left(f-\frac{n}{T_{f}}\right)\right)$

$+\frac{1}{T_{f}}\left(\frac{1}{N_{c}} \sum_{i=0}^{N_{c}-1}\left|X(f) \cdot\left(e^{-j 2 \pi f f_{c}}\right)^{i}\right|^{2}-\frac{1}{N_{c}^{2}}\left|\sum_{i=0}^{N_{c}-1} X(f) \cdot\left(e^{-j 2 \pi T_{c}}\right)^{i}\right|^{2}\right)$

$=\frac{1}{N_{c}^{2} T_{f}^{2}} \cdot \sum_{n=-\infty}^{+\infty}\left(\mid X\left(\frac{n}{T_{c}}\right)^{2} \cdot \delta\left(f-\frac{n}{T_{c}}\right)\right)+\frac{1}{T_{f}}|X(f)|^{2} \cdot\left(1-\frac{1}{N_{c}^{2}}\left|\sum_{i=0}^{N_{c}-1}\left(e^{-j 2 \pi T_{c}}\right)^{i}\right|\right)$

As seen from the above example, the lines are not completely eliminated by the use of a long spreading sequence. The distance between two adjacent spectral lines is inversely proportional to the chip duration. Increasing $N_{c}$ would only put the spectral lines further apart. If $N_{c}$ goes to infinity, which is equivalent to a uniform distribution of the pulse over the frame, all spectral lines occur at infinite spacing and thus effectively vanish. Practical considerations usually prevent the use of infinite $N_{c}$ in UWB systems. The generation of TH positions via PN sequences, or other digital codes, or from stored tables, requires a quantization, and thus a finite number of possible positions $N_{c}$. Ref. [6] also noted that some spectral lines cannot be reduced by designing a $\mathrm{TH}$ sequence for example when $T_{f} / T_{c}=m / n$ where $m$ and $n$ are relatively prime numbers.

\section{Symbol-based polarity randomization}

\section{A The basic idea of polarity randomization}

As mentioned above, the spectral lines are a serious obstacle for efficient implementation of UWB systems. We therefore suggest a simple but effective method to eliminate them: randomizing the polarity of the transmitted pulses. In other words, Eq. (1) is replaced by

$s_{t r}(t)=\sum_{j=-\infty}^{\infty} p_{j} \cdot x\left(t-j T_{f}-c_{j} T_{c}-b_{\left[j / N_{f}\right\rfloor} \Delta\right)$

where $p_{j}$ is a pulse randomization sequence. This method which we proposed in our VTC2003 paper [17], has since been generally accepted and widely used in the literature. We will 
show in the following that the sequence eliminates spectral lines under all considered circumstances but that, depending on the specific properties of the TH sequence, the continuous part of the PSD has different shapes.

\section{B Symbol-based polarity randomization with short TH sequence}

The first case we consider randomizes the polarity (sign) of the transmitted pulses on a symbol-by-symbol basis, where we assume a short $\mathrm{TH}$ sequence. This has a formal similarity to joint PPM/PAM as modulation format (combined with $\mathrm{TH}$ for multiple access). The difference is that in our case, the sign of the transmit pulses does not bear any information, and can be easily discarded by simplified receiver structures (envelope detectors). The introduction of the pseudorandom polarity serves only to improve the spectral properties of the transmit signal. As we change randomly the polarity of the $\mathrm{M}$ symbols $s_{i}$; the signal can be viewed as a pulse train composed of $2 \mathrm{M}$ antipodal symbols. Thus, Eq. (2) can be rewritten as the PSD of a signal with antipodal modulation:

$$
\begin{aligned}
G_{s}(f)= & \frac{1}{M^{2} T_{s}^{2}} \cdot \sum_{n=-\infty}^{+\infty}\left(\left|\frac{1}{2} \sum_{i=0}^{M-1} S_{i}\left(\frac{n}{T_{s}}\right)+\frac{1}{2} \sum_{j=0}^{M-1} S_{j}\left(\frac{n}{T_{s}}\right)\right|^{2} \delta\left(f-\frac{n}{T_{s}}\right)\right) \\
& +\frac{1}{T_{s}}\left(\sum_{i=0}^{M-1} \frac{1}{M} \cdot\left|S_{i}(f)\right|^{2}-\left|\frac{1}{M} \sum_{i=0}^{M-1} \frac{1}{2} S_{i}(f)+\frac{1}{M} \sum_{j=0}^{M-1} \frac{1}{2} S_{j}(f)\right|^{2}\right)
\end{aligned}
$$

where $S_{i}$ is the Fourier Transform of the $\mathrm{i}^{\text {th }}$ shifted version of the short TH sequence of pulses (not a single pulse!). The symbols $\mathrm{s}_{\mathrm{i}+\mathrm{M}}$ are the symbols $\mathrm{s}_{\mathrm{i}}$ with an opposite polarity; thus, $S_{i+M}=-S_{i}$ for i from 0 to $\mathrm{M}-1$. The spectrum then becomes $G_{s}(f)=\frac{1}{M \cdot T_{s}} \sum_{i=0}^{M-1}\left|S_{i}(f)\right|^{2}$

Thus, the spectral lines disappear [31]. Furthermore, from Eq. (8), we see that the spectrum of the signal is defined by the summation of the spectrum of the symbols.

From Eq. (8), it is also clear that the same statement holds for the different modulation formats, like PPM and on-off keying. Furthermore, it is not required anymore to have equiprobable symbols to control the spectral characteristics of the modulated signal by removing the spectral lines. Even if the original modulated signal has non-equiprobable symbols or non-antipodal modulation schemes, the symbol polarity scrambling solves these two issues by creating pairs of independent equiprobable waveforms of opposite polarity. This is a sufficient condition to eliminate spectral lines.

For binary PPM four modulation symbols need to be taken into account: one symbol coding for ' 0 ', one shifted symbol coding for ' 1 ' and the same two symbols with opposite polarity. For the case of OOK, we obtain the PSD in a similar form: the modulation now has 3 different symbols $(1,-1,0)$ of probability $1 / 4,1 / 4$, and $1 / 2$. The spectral lines vanish and the PSD is only given by the basic signaling waveform (Fig. 4). [31]

Thus, changing randomly the polarity from symbol to symbol (symbol-based polarity randomization) provides an efficient way to eliminate spectral lines. The continuous part of the PSD of the signal is determined by the "symbol waveform", i.e., the sequence of time-shifted basis pulses. In other words, the PSD of the signal is the multiplication of the PSD of the pulse and the PSD of the TH sequence. However, the TH sequence creates peaks in the spectrum that necessitate a

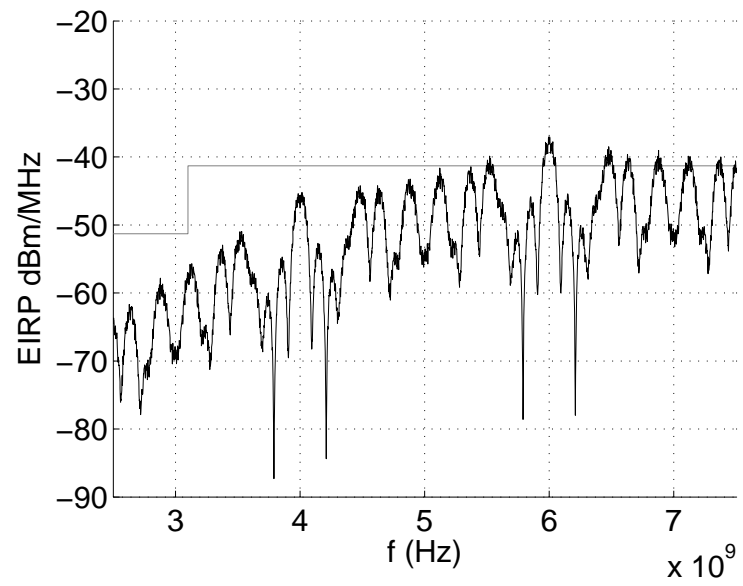

Fig. 4: PSD of a signal with the TH sequence given Fig. 1 for BPSK modulation, OOK or 2PPM with symbol based polarity randomization.

reduction of the total transmit power. Similar to the wellknown "Crest factor" in the time domain, we will call the peakto-average ratio of the PSD the "spectral crest factor". Figure 4 shows the spectrum of a signal with the TH sequence defined in Sec. II.C: the spectral crest factor is $5 \mathrm{~dB}$.

The polarity randomization also offers the possibility of shaping the spectrum. The design of a TH hopping sequence in order to place spectral notches is possible, for example by optimizing the TH sequence. However, finding a large set of TH sequences (required for multiple access) that fulfill both good cross-correlation properties and have specific properties of the PSD is a difficult problem and generally requires extensive methods to refine and optimize these sets.

The expression of the PSD when BPSK is used as modulation format is similar to the PSD obtained with PPM, but with $\Delta=0$. The polarity of the symbols is affected by the modulation, and thus information-carrying. 5

One might wonder why PPM is considered at all in combination with polarity hopping. After all, the transmitter of polarity-hopped PPM is more complicated than for BPSK alone, and BPSK provides better BER at the same SNR. However, PPM provides for a simplified receiver implementation, since the polarity of the received signal can be ignored, and a pure energy detection can be performed. Thus, both modulation formats have their areas of application.

\section{C Symbol-based polarity randomization with long $\mathbf{T H}$ sequence}

In many cases, the shaping of the spectrum, and especially the spectral ripples that occur even in such a shaped spectrum, is undesirable. The goal is a signal of which the PSD is as smooth as possible, and ideally identical to the PSD of the basic pulse, e.g., our single Gaussian pulse. In this section, we describe one method to smooth the spectrum by combining short TH sequences with good spectral characteristics.

The basis for this approach is that a long TH sequence can be thought of as a sequence of short TH sequences. In other

5 An additional polarity randomization can be superimposed to ensure zero-mean signals even if the underlying data are not equiprobable. 


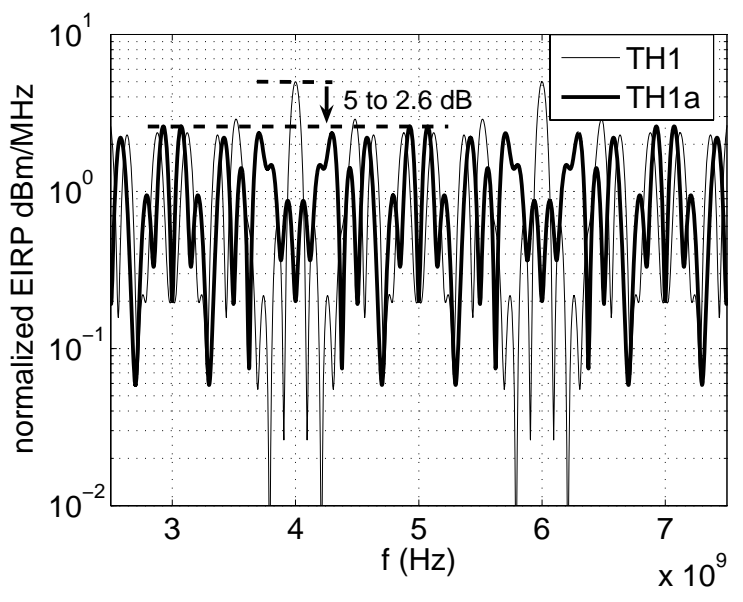

Fig. 5: effect of a short pulse based polarity randomization on the normalized PSD of TH1

words, the waveform of a symbol is taken randomly from a preselected set of waveforms and its polarity is then scrambled by the symbol-based polarity randomization sequence. We have seen with Eq. (8) that the spectrum is then the average of the squared FT of each symbol. If the peaks of their respective PSDs occur at different frequencies, the total peak to average ratio will be reduced.

We also find that the symbol-based polarity randomization eliminates the cross terms (second term of the continuous part of the spectrum Eq. (2)) and thus simplifies the design of the spectrum: the design of the waveforms can be done one by one, adjusting the spectral characteristic of each of them in order to control the PSD of the signal, which is simply their average. If the crossterms would not vanish, all waveforms would have to be optimized jointly.

The spectrum can be formulated mathematically as

$$
G_{s}(f)=\frac{1}{N_{c}^{N_{f}} . T_{s}}|X(f)|^{2} \cdot \sum_{i=0}^{N_{c} N_{f}-1}\left|\sum_{l=0}^{N_{f}-1} e^{-j 2 \pi f\left(l . T f+r_{i l} \cdot T_{c}\right)}\right|^{2}
$$

The use of several short $\mathrm{TH}$ sequences taken randomly for different symbols helps smoothing the spectrum but does not necessarily reduce the spectral Crest factor. The problem is similar to the remaining spectral lines in the PSD of Section II.D when $\mathrm{m}$ and $\mathrm{n}$ are relatively prime numbers. A small number of high amplitude frequencies remain because of the frame structure of the signal. While it is possible to move those peaks to frequencies where they have less negative impact (e.g., frequencies where the constituent pulses provide a smaller spectral density), such signal is complex. The following section proposes a better solution to this problem.

\section{Pulse-based polarity randomization}

The use of long $\mathrm{TH}$ sequences is often undesirable. In particular, it makes synchronization more difficult, and complicates the design of equalizer schemes.

For this reason, we propose to modify the symbol structure by randomizing the polarity of each transmitted pulse and without changing the pulse positions. Thus, the variables $p_{j}$ in Eq. (6) become i.i.d. random variables that can take on the values +1 and -1 .

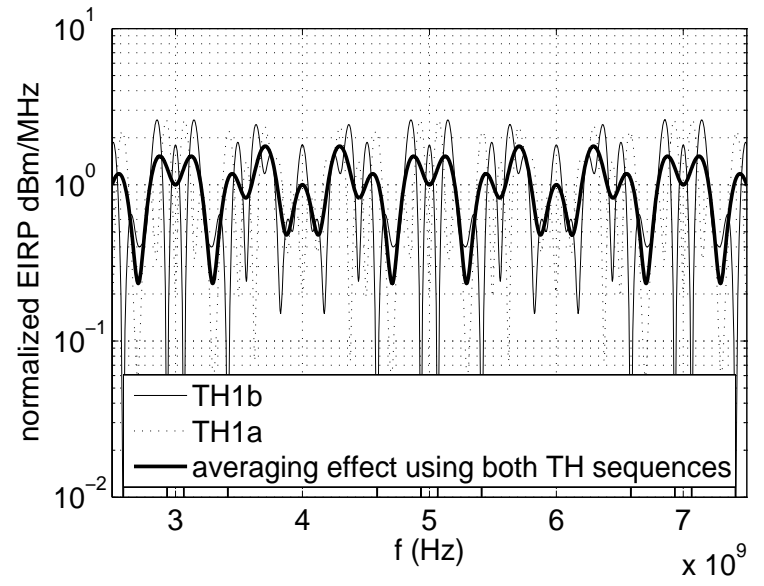

Fig. 6: effect of a long pulse based polarity randomization on the normalized PSD of TH1

\section{A Short TH sequence and short pulse-based polarity randomization sequence}

A short polarity randomization sequence is a sequence that is the same for all transmitted symbols. Thus, also the combination of $\mathrm{TH}$ sequence and polarity randomization sequence is repeated with the symbol period. In other words, the transmit signal is a sum of modulated "basic signal" waveforms, the basic waveform, consisting of a number of delayed pulses with different polarities, is chosen once but then remains fixed.

The situation is thus similar to Sec. II.C, though the TH sequence defined there (TH1) only contained pulses with the same polarity. In the case of pulse-based polarity randomization, the PSD is again given by Eq. (8), but now the "basic signal" waveforms have a different, more flexible structure, i.e., the pulses can have different polarities. This flexibility helps to better design the spectrum of the signal to reduce the spectral crest factor due to the $\mathrm{TH}$ structure and limits the power back off.

As an example, we consider again TH1, $c_{j}=\left(\begin{array}{lllll}1 & 0 & 0 & 1 & 1\end{array}\right)$, but now in conjunction with the short pulse-based polarity randomization sequence $p_{j}=\left(\begin{array}{lllll}-1 & 1 & -1 & 1 & 1\end{array}\right)$. We call this new sequence TH1a. In order to focus on the spectral characteristics of the TH sequences, figure 5 shows the PSD of TH1 and TH1a without the contribution of the envelope $S(f)$ due to the pulse. The comparison of these normalized PSDs with and without the pulse-based randomization shows that inverting the polarity of some pulses can reduce significantly the spectral crest factor of the PSD ( $5 \mathrm{~dB}$ for TH1 versus $2.6 \mathrm{~dB}$ for TH1a).

A further generalization is possible by using a short $\mathrm{TH}$ sequence in conjunction with a long (pulse-based) polarity randomization sequence. As mentioned in section III B, the symbol-based polarity randomization allows to simply average the spectral characteristics of several waveforms.

A random selection among several short pulse-based polarity randomization sequences, using the same $\mathrm{TH}$ sequence, can thus reduce the crest factor.

For example, using TH1a and TH1b defined by $c_{j}=\left(\begin{array}{lll}1 & 0 & 0\end{array}\right.$ $11)$ and the short pulse-based polarity randomization sequences $p_{j}=\left(\begin{array}{lllll}-1 & 1 & -1 & 1 & 1\end{array}\right)$ and $p_{j}=\left(\begin{array}{lllll}1 & -1 & 1 & 1 & 1\end{array}\right)$, respectively, 


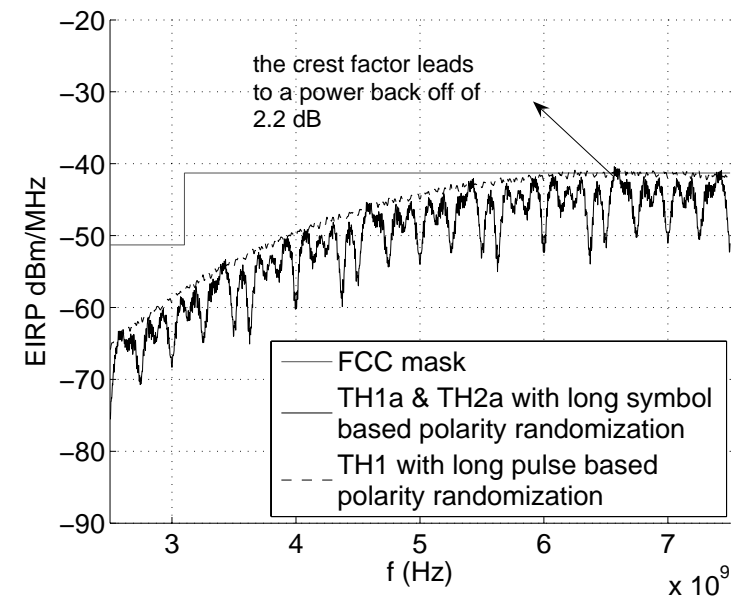

Fig. 7: effect of short \& long pulse-based random polarity

reduces the crest factor by $0.83 \mathrm{~dB}$ (Fig. 6) compared to the use of TH1a only..

Another possibility to flatten the spectrum is the use of a pulse-based short polarity randomization sequence, together with a symbol-based polarity randomization and a long $\mathrm{TH}$ sequence. This allows an optimization of the envelop of the PSD similar to the discussion in Sec. III.C; however, we now can now better optimize the PSD of the constituent waveforms (due to the larger number of degrees of freedom for creating those waveforms, as discussed earlier in this section). In Fig. 7 we use randomly the sequences TH1a and TH2a $\left(C_{j}=\left(\begin{array}{llll}0 & 1 & 0 & 1\end{array}\right.\right.$

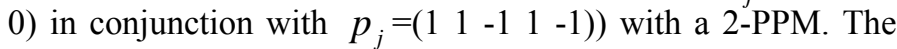
crest factor is about $0.5 \mathrm{~dB}$ better than using one of these sequences alone. We emphasize the fact that, due to the symbol-based polarity randomization, the spectrum of Fig.7 is the same for OOK, 2-PPM and BPSK.

\section{B Long TH sequence and long pulse-based polarity randomization sequence}

This case is a generalization of the symbol-based polarity randomization. Randomizing the polarity per pulse allows a description of the signal with independent, equiprobable and antipodal symbols defined over a frame duration. Having $\mathrm{Nc}$ possible positions per frame and a 2-PPM, the possible number of different frames is $2.2^{N_{c}}$. We note that the antipodal modulation has a constellation of $2^{N_{c}}$ symbols for BPSK and $2^{N_{c}}+1$ for OOK $(+1$ because of the null symbol). For a $2-$ PPM, Eq. (8) leads to [31]

$$
G_{s}(f)=\frac{1}{T_{s}}|X(f)|^{2}
$$

Eq. (9) shows that the use of a longer code to scramble the polarity of each pulse of the TH sequence allows us to get the spectrum of the original waveform. This general form shows that a DS or TH system with a long pulse-based polarity scrambler eliminates the spectral lines and reduces the need of a power back off due to the peak to average ratio caused by the structure of a short TH code. The spectrum of a PPM, OOK, or BPSK signal using this long scrambling code is given by the waveform of the pulse and is identical to the dashed one given in fig. 7.

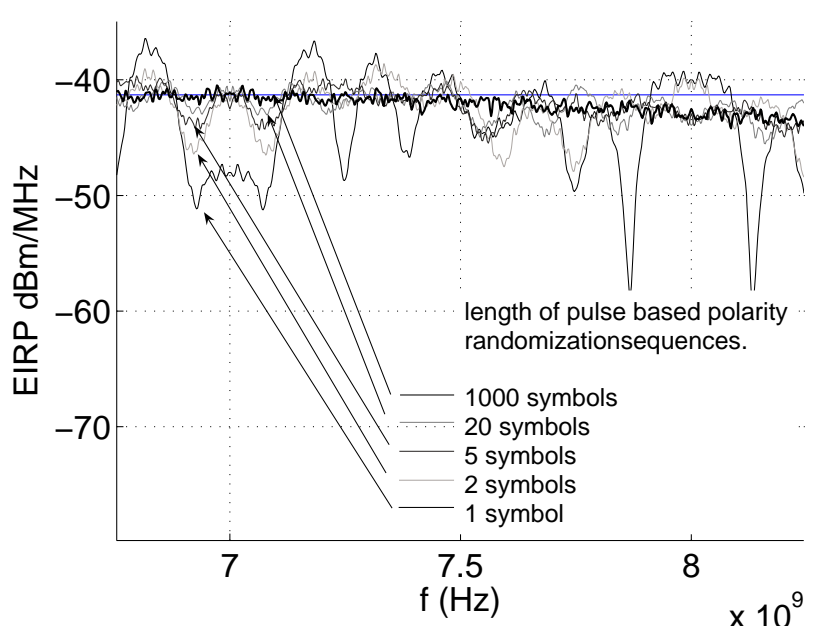

Fig. 8: Effect of the length of the pulse based polarity randomization sequence on the PSD of a BPSK signal with short TH sequence.

Finally, we note that the result remains valid if the positions of the pulses are not restricted to a structure with frames. This is the case for example when a jitter is considered to randomize the position of the pulses. The PSD of the signal is given by the pulse shape. We emphasis that this result also holds if the time hopping is short and the polarity randomization sequence is long.

In this paper, a complete randomization of the polarity when considering symbol-based or pulse-based polarity randomization was assumed. We checked by simulation that a finite sequence provides similar results. Fig. 8 illustrates the smoothing of the spectrum and the reduction of the Crest factor when the length of the pulse-based polarity randomization sequence changes from one symbol duration to one thousand. A short TH sequence is used and the modulation format for this simulation is BPSK. The results are similar for PPM and OOK with a long symbol-based polarity randomization sequence. Simulations with a long TH sequence provide qualitatively similar results [31].

The FCC regulations require a certain power spectral density within each $1 \mathrm{MHz}$ band. Thus, fine structures of the spectrum within this bandwidth do not have an impact on the compliance with the FCC regulations. Hence, any periodicity that is significantly larger than $10^{-6} \mathrm{~s}$ can be ignored.

\section{V.Conclusion}

This paper discussed the influence of the modulation format, multiple access format, and pulse shape on the spectral characteristics of impulse radio. We showed that PPM and time hopping lead to similar spectral characteristics, namely spectral lines. We suggested a remedy to solve this problem:

- Randomization of the polarity of the transmitted symbols or pulses eliminates the spectral lines

- Polarity randomization on a symbol-by-symbol basis, combined with the use of several short TH sequences or a long $\mathrm{TH}$ sequence can lead to the reduction of spectral crest factors.

- Polarity randomization on a pulse-by-pulse basis with a short sequence, combined with a short TH sequence, 
allows to better design the spectrum and greatly reduces the spectral crest factor.

- The use of long pulse-based polarity randomization codes ensures that the spectrum of the composite signal is equal to the spectrum of the constituent pulse.

The last case (spectrum of the signal identical to the spectrum of the underlying pulse) is desirable for practical applications. Shaping this waveform in order to get a better power efficiency or to reduce interference and get better coexistence with other systems such as $802.11 \mathrm{a}$ can then be achieved without any further consideration for the modulation scheme. It is possible to design the PSD of the UWB signal using the results of [26] that considers shaping a waveform to avoid interference and [27] that gives general methods to shape the spectrum.

This paper has concentrated on the spectral properties of the transmitted signal which are, however, not the only consideration in the signal design. While the use of long spreading sequences (either time-hopping or polarity randomization) simplifies the spectrum design, it complicates receiver design. Furthermore, the existence of a sufficiently large ensemble of hopping sequences with appropriate cross correlation properties is important for the multiple-access capabilities of the system. Nevertheless, in many cases, improving the received SNR, and thus maximizing the output power within the limits of the FCC, is an overriding concern. Our results allow a better exploitation of the limited resource "transmit power".

\section{References}

[1] R.A. Scholtz, "Multiple access with time-hopping impulse modulation", IEEE Military Communications Conference 1993 Volume 2, MILCOM '93, Conference record 'Communications on the Move', 11-14 Oct. 1993, p447-450 [2] M.Z. Win, R.A. Scholtz, "Impulse radio: how it works", IEEE Communications Letters Volume 2, Issue 2, Feb. 1998, p36-38

[3] M.Z. Win, R.A. Scholtz, "Ultra-wide bandwidth time-hopping spreadspectrum impulse radio for wireless multiple-access communications", IEEE Transactions on Communications Volume 48, Issue 4, April 2000, p679-689

[4] A. Batra et al., "Multi-band OFDM physical layer proposal", Document IEEE 802.15-03/267r2, 2003

[5] J. McCorkle et al., "XTreme Spectrum CPF document", Document IEEE $802.15-03 / 154 \mathrm{r} 0,2003$

[6] R.C. Qiu, H. Liu, X. Shen, "Ultra-wideband for multiple access communications", IEEE Communications Magazine Volume 43, Issue 2, Feb 2005, p80-87

[7] L. Yang, G.B. Giannakis, "Ultra-wideband communications: an idea whose time has come", IEEE Signal Processing Magazine Volume 21, Issue 6, Nov. $2004, \mathrm{p} 26-54$

[8] M. G. di Benedetto et al. (ed.), "UWB communications systems - a comprehensive overview", EURASIP publishing, 2005.

[9] FCC 00-163, "Notice of Proposed Rule Making", May 2000

http://hraunfoss.fcc.gov/edocs_public/attachmatch/FCC-00-163A1.pdf

[10] FCC 02-48, "First Report and Order", April 2002

http://hraunfoss.fcc.gov/edocs_public/attachmatch/FCC-02-48A1.pdf

[11] S.G. Wilson, "Digital Modulation and Coding", 1996 edition, 4-PPM, p237; Pulse Amplitude Signaling, p238

[12] J.M.H. Elmirghani, R.A. Cryan, F.M. Clayton, "On the spectral estimation and synchronization of the cyclostationary optical fibre PPM process", IEEE Transactions on Communications Volume 43, Issue 234, Feb-Mar-Apr 1995, p1001-1012

[13] M.Z. Win, "Spectral density of random time-hopping spread-spectrum UWB signals with uniform timing jitter", IEEE Military Communications Conference Proceedings 1999 Volume 2, MILCOM 1999, 31 Oct.-3 Nov. 1999, p1196-1200

[14] M.Z. Win, "Spectral density of random UWB signals", IEEE Communications Letters Volume 6, Issue 12, Dec. 2002, p526-528

[15] J. Romme, L. Piazzo, "On the power spectral density of time-hopping impulse radio", IEEE Conference on Ultra Wideband Systems and Technologies 2002, Digest of Papers, 21-23 May 2002, p241-244
[16] L. Piazzo, J. Romme, "Spectrum control by means of the TH code in UWB systems", The 57th IEEE Semiannual Vehicular Technology Conference 2003 Volume 3, VTC 2003-Spring, 22-25 April 2003, p1649-1653

[17] Y.-P. Nakache; A.F. Molisch, "Spectral shape of UWB signals - influence of modulation format, multiple access scheme and pulse shape", The 57th IEEE Semiannual Vehicular Technology Conference 2003 Volume 4, VTC 2003Spring, 22-25 April 2003, p2510-2514

[18] D. Cassioli, F. Mazzenga, "Spectral analysis of UWB multiple access schemes using random scrambling", IEEE Transactions on Wireless Communications Volume 3, Issue 5, Sept. 2004, p1637-1647

[19] G.M. Maggio, N. Rulkov, L. Reggiani, "Pseudo-chaotic time hopping for UWB impulse radio", IEEE Transactions on Circuits and Systems I: Fundamental Theory and Applications Volume 48, Issue 12, Dec. 2001, p14241435

[20] S.G. Wilson, "Digital Modulation and Coding", 1996 edition, DSSS for CDMA, p248-250

[21] A. Saidi, "Spectrum Shaping Using Code-Hopping CDMA", the MITRE corporation, October 2004

http://www.mitre.org/work/tech_papers/tech_papers_04/04_1042/04_1042.pdf [22] S.G. Wilson, "Digital Modulation and Coding", 1996 edition, Power Spectrum for General Memoryless Modulation, p235-236

[23] H. Sheng, P. Orlik, A.M. Haimovich, L.J. Cimini Jr., J. Zhang, "On the spectral and power requirements for ultra-wideband transmission", IEEE International Conference on Communications 2003 Volume 1, ICC'03, 11-15 May 2003, p738-742

[24] A.F. Molisch,Y. G. Li, Y.-P. Nakache, P. Orlik, M. Miyake, Y. Wu, S. Gezici, H. Sheng, S.Y. Kung, H. Kobayashi, H.V. Poor, A. Haimovich, J. Zhang, "A Low-Cost Time Hopping Impulse Radio System for High Data Rate Transmission", EURASIP Journal on Applied Signal Processing, JASP, 2005, p397-412

[25] M.-G. Di Benedetto, G. Giancola, "Understanding Ultra Wide Band Radio Fundamentals", Chapter 3, part 2: The PPM-TH-UWB Case, Prentice Hall PTR, 1st Ed., Jun 17 2004, p107

[26] C.J. Le Martret, G.B. Giannakis, "All-digital PAM impulse radio for multiple-access through frequency-selective multipath", IEEE Global Telecommunications Conference 2000 Volume 1, GLOBECOM '00, 27 Nov.-1 Dec. 2000, p77-81

[27] Y. Wu, A.F. Molisch, S.Y. Kung, J. Zhang, "Impulse Radio Pulse Shaping for Ultra-Wide Bandwidth UWB Systems", Proc. IEEE Int. Symp. Personal Indoor Mobile Radio Comm 2003, p877-881.

[28] J. Bellorado, S.S. Ghassenzadeh, A. Kavcic, B. Tarokh, V. Tarokh, "Timehopping sequence design for narrowband interference suppression”, IEEE 60th Vehicular Technology Conference 2004, Volume 6, VTC2004-Fall, 26-29 Sept., p3925-3929

[29] I. Guvenc, H. Arslan, "TH-sequence construction for centralised UWB-IR systems in dispersive channels", Electronics Letters, Volume 40, Issue 8, 15 April 2004, p491-492

[30] Z. Zhang, F. Zeng, L. Ge, "Construction of multiple-stage time-hopping sequences in time-hopping spread spectrum ultra wideband", IEEE 60th Vehicular Technology Conference 2004 Volume 2. VTC2004-Fall, 26-29 Sept., p832-836

[31] Y.-P. Nakache, A.F. Molisch, "PSD of UWB signals", TR2005-113, 2005, http://www.merl.com/publications/TR2005-113/TR2005-113.doc.

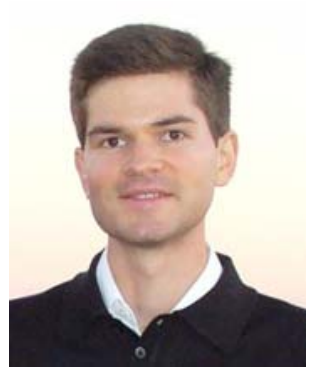

Yves-Paul Nakache received the M.S. degree from the Ecole Superieure d'Ingenieurs en Electronique et Electrotechnique de Paris (ESIEE Paris) in 2000. He received his B.S. degree in Physics, concurrently, from the University Paris VII in 1998. In 2000 his research activity focused on a very low-bit encoding of prosody for a speech vocoder with Thales Communication for the RNRT Project SYMPATEX. Then he joined Mitsubishi Electric Research Laboratories. His current research interests include spectral shaping for impulse radio ultra-wideband (UWB) transceivers, spreading techniques for MB-OFDM systems, low complexity UWB transceivers, and modulation format for noncoherent receivers.

Andreas F. Molisch, for recent photo and bio see, e.g., IEEE Trans. Wireless Comm., 4, p.154 (2005). 